\section{Molecular Modelling for Beginners}

by Alan Hinchcliffe (ed): Wiley- $\mathrm{VCH}$, Weinheim, 2nd edn, 2008, pp 428, ISBN 978-0-470-51313-2.

Price: EUR 129.00, USD 170.00

This book has been written as an introduction to molecular modelling and is particularly useful to students new to the field. It is particularly good as a reference material as it explains many commonly used terms and equations in a clear and concise manner. The author covers a wide range of the basic principles of molecular modelling which gives a good base for more advanced topics. There are 24 chapters with each chapter divided into subsections with a short introduction followed by definitions, terminology, theory and problem sets.

The first three chapters serve as an introduction to the principles behind molecular modelling. Readers should not be put off by the large number of equations in the opening chapters as these are very well thought out and a great introduction to the terminology used in later chapters. Chapter 4 continues with a general introduction to molecular mechanics and a discussion of some commercially available force fields. Chapter 5 has a more detailed discussion of potential energy surfaces and Chapter 6 gives some examples. Chapters 7 through to 10 give an introduction and background to molecular dynamics simulations moving from Monte Carlo modelling of thermodynamic properties to molecular dynamics and studying time evolution within a system.

Chapter 11 introduces quantum modelling starting from the Schrödinger equation and working through Chapters 12 and 13 covering topics such as the Pauli exclusion principle, Bohr's theory, and Dirac theory of the electron. Chapter 14 introduces the Hartree-Fock model with
Chapters 15-17 extending to the HFLCAO model and giving some examples. Semi-empirical models are discussed in some depth in Chapter 18. Chapter 19 deals with electron correlation and Møller-Plesset perturbation theory moving onto Density Functional Theory in the next chapter. Chapters 21-23 look at some practical applications such as gaining accurate thermodynamic properties, transition states, and dealing with solvent effects. The last chapter turns its attention to the increasingly important QM/MM approach to large systems.

In summary, I certainly feel that this would be a very useful book to have in any library as it covers a wide range of modelling methodologies and more importantly their theoretical basis. It is particularly suitable as reference material for a teaching course as the problem set and mathematical appendix are an excellent resource.

A. Trewin 